\title{
Reservoir based on Intelligent Monitoring and Forecasting System
}

\author{
Yu Lin ${ }^{1, a^{*}}$, Zhiwei Lin ${ }^{2}$ and Jing Tian ${ }^{3}$ \\ ${ }^{1}$ The Department of Computer \& EEE Oxbridge College, Kunming University of Science and \\ Technology, Kunming, China \\ ${ }^{2}$ Faculty of Civil Engineering and Mechanic, Kunming University of Science and Technology, \\ Kunming, China \\ ${ }^{3}$ Yunnan computer software technology development research center, Kunming, China \\ akmly2011@163.com
}

Keywords: GPRS; ZigBee; Modbus; Wireless transmission; Intelligent control

\begin{abstract}
Reservoir undertakes a role in flood control and storing water in flood, regulating peak; Dry season it undertakes to provide water for urban and agricultural functions. The most reservoirs of our country construct in fifty and sixty times, mainly to small and medium sized. Hydrological monitoring system is widely used at large reservoirs, however it is not yet widely used for small reservoirs due to small capital, technology and other aspects. In the rainy season, the reservoir is limited by the impact of flood control level required to maintain enough capacity to prevent sudden flood, therefore it will lead to be unable to fulfill the target of maximum capacity at the end of the flood season. Dynamic flood control level of application is subject to historical information, geographical constraints and theoretical calculation and so on. The most important factor of the above is whether the reservoir managers timely are informed of the basin hydrological data. The paper focuses on automated acquisition, reliable transmission data, PC software for intelligent data analysis and timely warning, spending on monitoring hydrological data of the reservoir basin spend less than before.
\end{abstract}

\section{Introduction}

Most of China's water conservancy facilities are established during the Cultural Revolution, the age of them nearly 50 years, and the presence of reservoirs runs a risk. Although the state attaches great importance to invest a lot of money for reservoir reinforcement to avoid risk each year, along with climate change and the large number of reservoirs, China Southern and Northern differences between the dry and rainy season rainfall. Expansion of the city's demand for water is also growing. Faced with the contradiction in the flood season maximum storage and flood control, the security of the reservoir has become a question which should not be ignored. So accurate and reliable information forecasting system acts as an important safeguard and improves the safety factor of reservoir operation. If the danger appeared irresistible, it timely can send warning messages to people to reduce losses[1-2].

Automatic water regime forecasting and monitoring system using in a large, wide application of cascade reservoirs, the vast number of small reservoirs of information technology, low level of automation, following the traditional manual observation mode, existing large errors, dynamic flood control level should not be set to achieve the largest reservoir store when the rainy season ending. It needs a mature, reliable, reasonably priced, intelligent system of hydrological forecasting system to achieve the small reservoir hydrologic data collection, monitoring, analysis and processing to guarantee the safe operation of the reservoir[3].

\section{System Design}

Intelligent monitoring and forecasting system of hydrological reservoir must be designed to meet the country's design requirements of flood control system, so the system should meet the following requirements.

1) Simple structure, reliable performance, reliable operation in unattended condition. 
2) Working mode: automatic and manual reporting mode query, you can freely set mode.

3) The data collected can generate reports, facilitating managers queried. Considering the importance of the data, it can be able to regularly back up.

4) Reservoir control center receives hydrological data of drainage basin from monitoring stations in less than 30 minutes.

5) System should be designed to facilitate subsequent upgrades[4].

The system includes two subsystems: 1. Hydrological Data Acquisition System of Monitoring Stations; 2. Reservoir Control center. Considering the different frequencies of data reported in the flood season and non-flood, divided into two data acquisition modes, auto mode, half an hour once collected and reported; manual mode, when you need to read the data, clicking the "Manual Search" button, reservoir control center sending a set of Modbus protocol command. Data acquisition module is mainly constituted by a microcontroller which uses it reading the data of hydrological sensor transmitter. The data will be sent to the GPRS DTU and ZigBee DTU through RS-485 protocol after A / D conversion and reservoir control center finally receives data. The communication between monitoring stations and reservoir control center uses GPRS and ZigBee protocol, short- distance communication using ZigBee, long-distance communication using GPRS, that the two communications complement each other. Reservoir control center uses the ForceControl 7.0 to establish master-slave relation with each monitoring station via Modbus RTU to obtain data [5]. Reservoir control center could intelligently analyze and process data. If it is abnormal timely and accurate sending out alarm information, the ForceControl 7.0 as the core of the system exchanges data with each monitoring station, access to water level, displaying storage capacity and other data. It stores and analyzes the data from monitoring stations reported data then makes intelligent judgment. The system could timely and reliable send alarm information, if the measured value exceeds the set value. What the system works is shown in Fig. 1.

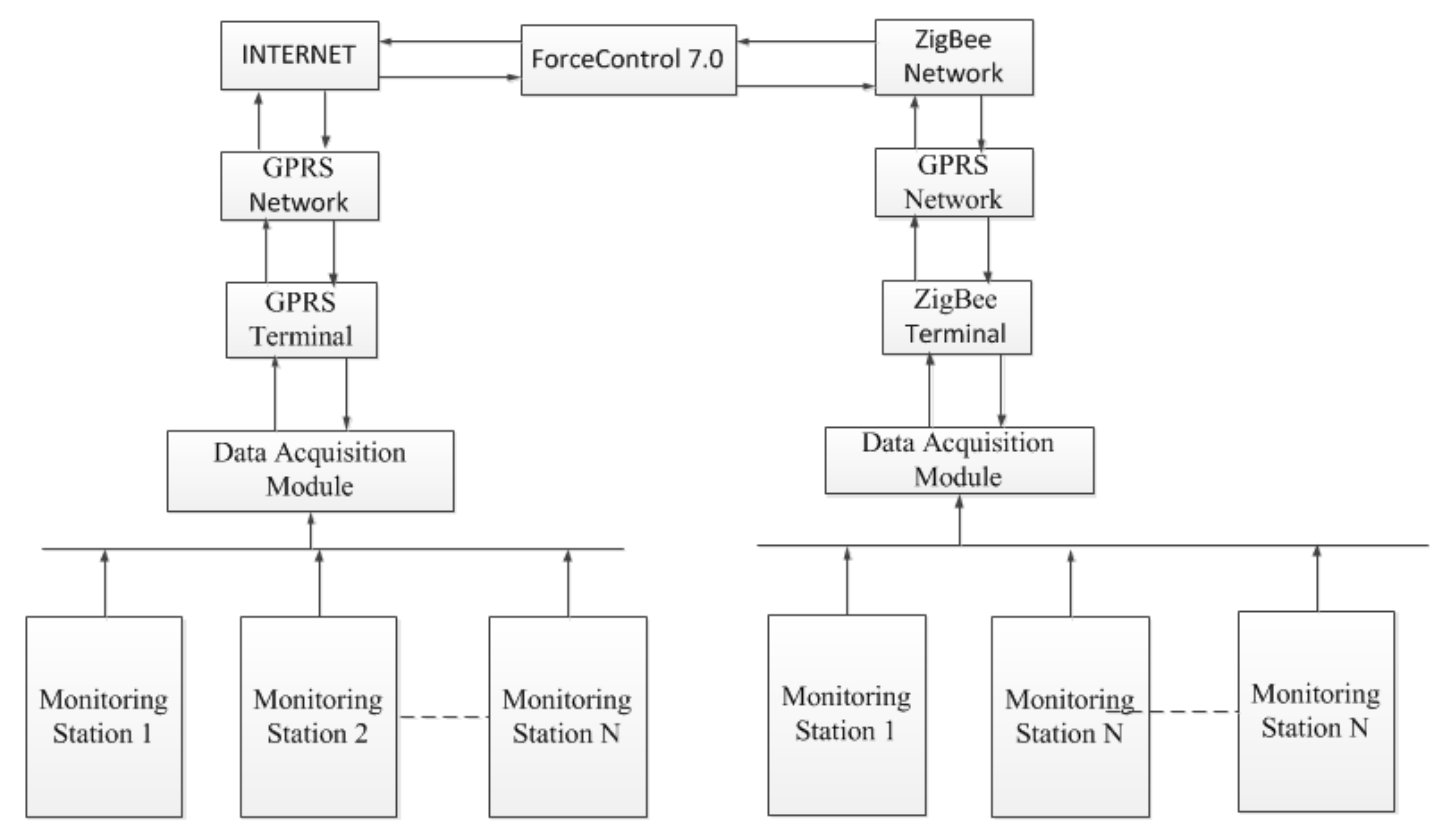

Figure 1. System working diagram

\section{Hydrological Data Report}

In order to distinguish different monitoring stations, system adopts the Modbus master-slave protocol, reservoir control center as the only one master station in the whole Modbus network. ForceControl 7.0 acts as master station, the remaining monitoring stations as a slave, and each monitoring has only one address in the whole Modbus network. Data acquisition module acquires data from hydrological 
sensor transmitter via RS485 with the program to convert it to Modbus command format then it transmits data to ForceControl 7.0 via ZigBee and GPRS [6].

In order to improve the scope, the system uses two types of data collection: 1.Automatically collection, microcontroller reads time from the clock chip to decide whether to read data comparing the setting time in advance, applying to non-flood season; 2.Manual collection, reservoir control center sends Modbus command via GPRS or ZigBee Monitoring station receives command and parses it comparing with the parameters which sets program in advance [7]. If the result is consistent, microcontroller starts to read the data of hydrological sensor transmitter and reports. The advantage of this way is real strong, good flexibility, suitable for frequent flood data collecting[8-10]. What the monitoring station works in manual mode is shown in Fig. 2.

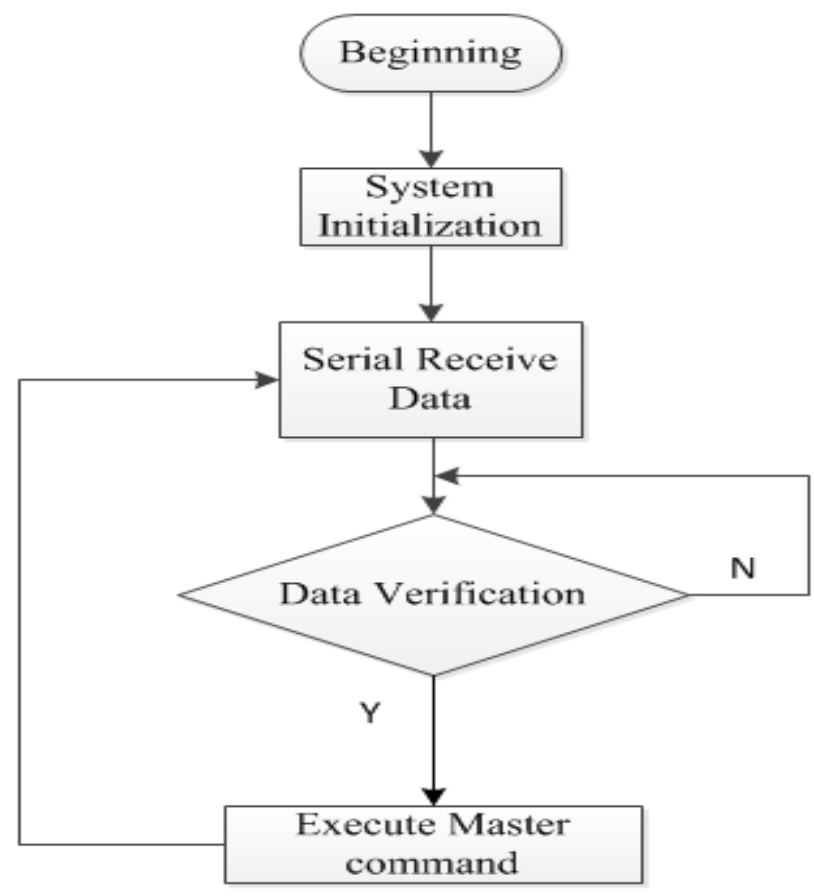

Figure 2. manual mode

\section{Hydrologic Data Calibration}

For the reason of water level data being susceptible to natural factors, the system needs a way of filtering to improve the accuracy of data collection. In a short time the water level change is slow, weighing several filtering methods, the system uses the "Arithmetic average filtering method".Within 1 second consecutive reading dozens of sampling data to calculate the average which is suitable for random noise signal filtering, the method can effectively filter out interference of the water level caused by the nature.

\section{System Operation and Testing}

The core of the paper for the reservoir is whether to achieve the goal of intelligent system operation: 1.Achieving accurate and timely data collection, displaying on the monitor screen; 2.Under exceptional condition it can alarm timely and reliable, at the same time accurately inform the staff where the fault comes from. Data collection is divided into two types: 1.Accord to set the time in advance to report data; 2. Click "Manual Search", system can obtain real-time data. Through the above tests to detect whether the system is working properly. System testing is shown in Fig. 3. 


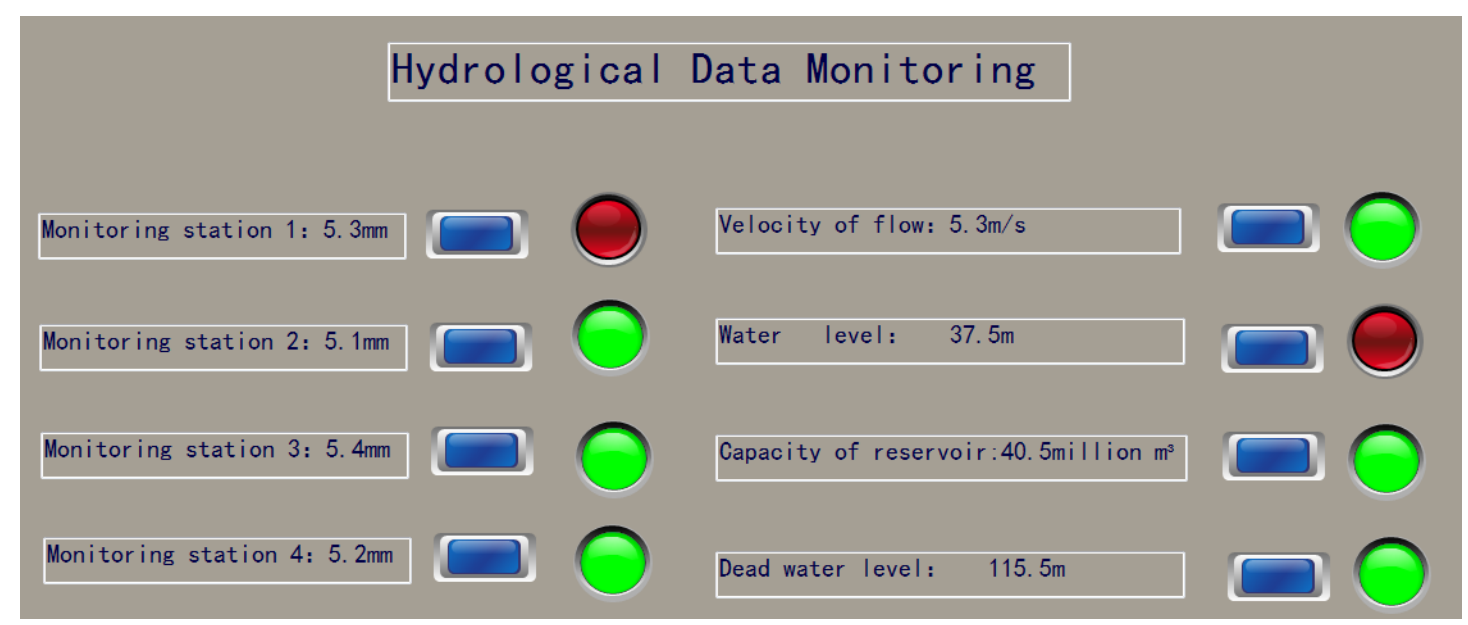

Figure 3. System testing

\section{Summary}

The paper bases on the intelligent reservoir hydrologic information system as an object and elaborates the system development and the key problems to solve. It combines with the development of communications, sensors, computers and other information technology to achieve hydrological data collection of the basin-wide reservoir, analysis, processing and intelligent hydrological forecasting. At the same time it improves the coefficient of the safe operation of the reservoir and reduces the operation cost and lay the foundation for future unmanned.

\section{References}

[1] Chengwu Xu. Calculation and management of water resources.Science Press, 2011, pp. 3-5.

[2] $\mathrm{ZuYu}$ Chen. Water Resources and Hydropower Engineering Risk Analysis and Reliability Design Technology Advances.China Water \& Power Press, 2009, pp. 1-3.

[3] Xuehong Sun, Jin Che, Cheng Zhang. The research of the water conservancy data acquisition and remote monitoring system based on GPRS. An hui Agricultural Sciences, 2009, Vol. 37(36), pp. 18057-18059.

[4] J.T.Devaraju, K.R.Suhas, H.K.Mohana, VA.Patil. Wireless Portable Microcontroller based Weather Monitoring Station.Measurement, 2015, pp. 76.

[5] Controller for real-time data collection and network communication based on Modbus/TCP in thermal power plant. Electric Power Automation Equipment, 2009, Vol. 08, pp. 128-131.

[6] Sushabhan Choudhury, Piyush Kuchhal, Rajesh Singh, Anita. ZigBee and Bluetooth Network based Sensory Data Acquisition System. Energy Conversion and Management, 2014, Vol. 88(12), pp. 49-59.

[7] Niv Goldenberg, Avishai Wool. Accurate modeling of Modbus/TCP for intrusion detection in SCADA systems. International Journal of Critical Infrastructure Protection, 2013, Vol. 6, pp. 63-75.

[8] Hao Zhang, Yanan Li, Huiling Zhu. Development for Protocol Conversion Gateway of Profibus and Modbus. Procedia Engineering, 2011, Vol. 15, pp. 767-771.

[9] Ping Ai. China Water Conservancy Information Assessment Study and Practice. Changjiang Press, 2011, pp. 1-5. 
[10]P Liu, GU Lichen, X Han, et al. Controller for real-time data collection and network communication based on Modbus/TCP in thermal power plant. Electric Power Automation Equipment, 2009, Vol. 08, pp. 128-131. 